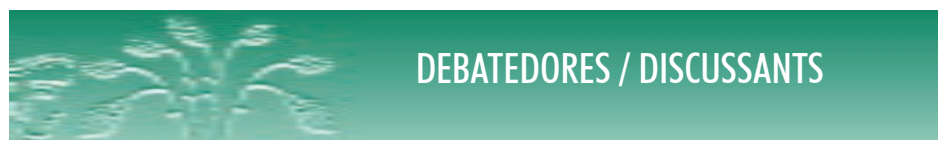

DOI: http://dx.doi.org/10.12957/demetra.2014.11791

\title{
Comentários dos autores
}

\section{Authors' comments}

\author{
Tatiane Nunes Pereira' \\ Kimielle Cristina Silva ${ }^{1}$ \\ Ana Carolina Lucena Pires' \\ Kelly Poliany de Souza Alves' \\ Ana Silvia Pavani Lemos² \\ Patricia Constante Jaime ${ }^{1}$
}

${ }^{1}$ Coordenação-Geral de Alimentação e Nutrição, Departamento de Atenção Básica, Secretaria de Atenção à Saúde, Ministério da Saúde. Brasília, DF, Brasil.

${ }^{2}$ Especialização em Saúde Coletiva e Educação em Saúde. Núcleo de Educação, Avaliação e Produção Pedagógica em Saúde. Faculdade de Educação. Universidade Federal do Rio Grande do Sul. Porto Alegre, RS, Brasil.

Correspondência / Correspondence Tatiane Nunes Pereira

Coordenação-Geral de Alimentação e Nutrição, Departamento de Atenção Básica, Secretaria de Atenção à Saúde, Ministério da Saúde. SAF SUL Quadra 02 Bloco E/F, Edifício Premium Torre II Piso Auditório (Subsolo) Sala 08. 70070-600 Brasília, DF, Brasil.

E-mail: tatiane.pereira@saude.gov.br
Perfil das demandas judiciais para fornecimento de fórmulas nutricionais encaminhadas ao Ministério da Saúde do Brasil

Inicialmente, gostaríamos de agradecer às debatedoras pelas contribuições, visto que seus comentários foram de extrema relevância para o aprofundamento das reflexões acerca da complexa temática abordada em nosso artigo. Ressaltamos a riqueza que olhares de diferentes atores trazem para a análise de um problema, por vezes, negligenciado tanto no campo da saúde, quanto da segurança alimentar e nutricional.

O artigo de Laura Araújo e Roseney Bellato traz a perspectiva do direito à saúde, garantido na Constituição Federal, com foco em crianças de até dez anos de idade, em função da maior demanda por fórmulas nutricionais advindas para este público, demonstrada em nosso artigo. Nesse sentido, reafirmamos nosso reconhecimento de que a judicialização é uma forma legítima de exigibilidade de direitos quando o Estado não provê insumos, ações e serviços de saúde essenciais à manutenção da vida. Concordamos com as afirmações das autoras de que tanto os responsáveis pelas crianças, quanto o Poder Judiciário agem em prol da garantia desse direito. $\mathrm{O}$ que questionamos em nosso artigo é a falta de rigor para a tomada de decisão judicial e todos os fatores envolvidos, como os conflitos de interesses, que podem existir e não são avaliados durante o julgamento. 
Assim, corroboramos as questões discutidas por Clarice Petramale, quando afirma que a falta de exigência de documentação do caso clínico e sua comprovação não dão segurança aos gestores de Saúde quanto à existência de benefícios ou possíveis malefícios com o uso do insumo solicitado, e que o fornecimento baseado somente na prescrição, e não no planejamento do cuidado integral, inviabiliza o controle quanto à melhor terapêutica, além de impossibilitar a programação orçamentária. Da mesma forma, a prescrição pela marca do produto dificulta a logística das compras e a garantia dos melhores preços para aquisição, além de deixar dúvidas quanto à isenção de envolvimento entre prescritores, advogados e associações de pacientes e produtores dos insumos.

Assim, como afirmado por Petramale, mostra-se necessário garantir que os processos para solicitação de fórmulas nutricionais explicitem cientificamente quais são os resultados esperados com o uso do insumo, em quanto tempo e como será monitorado o alcance dos resultados; e ainda, quais serão os critérios para alta e suspenção do tratamento, além de incluir os exames diagnósticos e avaliação do estado nutricional. Dessa forma, seria possível dar segurança ao próprio Poder Judiciário, quanto à sua decisão, e ao gestor da Saúde, possibilitando avaliação da necessidade e vulnerabilidade dos indivíduos solicitantes e garantindo que o fornecimento do insumo não seria eternizado sem justificativa. Assim, também contribuiria para a tomada de decisão quanto à incorporação do insumo como parte das ofertas terapêuticas disponíveis no SUS, evitando o uso de vias alternativas de acesso aos insumos.

As decisões individualizadas, desprezando os aspectos coletivos de distribuição de recursos às ações e serviços de saúde, podem evidenciar a tímida atuação do Judiciário em defesa do SUS e de sua operacionalização. Cabe questionar como vem se dando a participação do Poder Judiciário no apoio ao planejamento e à avaliação de políticas públicas de saúde no âmbito coletivo para além da atuação em casos individuais. Nesse sentido, reafirmamos o exposto em nosso artigo quanto à importância da comunicação efetiva e constante entre o Poder Judiciário e o Executivo, à qual adicionamos também a necessidade de apoio às instâncias de controle e participação social.

Nossa argumentação quanto à falta de critérios para tomada de decisão do Poder Judiciário, com aprovação de até 97,5\% das demandas, como demonstrado em diversos estudos, ${ }^{1-6}$ não significa que não reconhecemos que também há tensões e conflitos, além de captura por interesses privados, no âmbito do Poder Executivo. No campo da Alimentação e Nutrição, já foi demonstrado que as corporações utilizam diversas estratégias para influenciar políticas públicas, desde financiamento de pesquisas à realização de lobbies junto aos gestores e profissionais envolvidos com o tema. ${ }^{7}$ 
O Brasil é um dos poucos países que possuem sistema universal e gratuito de saúde, mas ainda existem limitações financeiras e organizacionais que impactam a efetivação de seus princípios de universalidade e equidade no acesso e integralidade do cuidado. Nesse sentido, concordamos que existem falhas no Sistema Único de Saúde (SUS), como exposto tanto por Laura Araújo e Roseney Bellato, quanto por Ligia Bahia.

É preconizado que o sistema seja organizado em Redes de Atenção à Saúde (RAS), que possuam a atenção básica como coordenadora do cuidado e ordenadora da atenção; no entanto, ainda prevalece o modelo hospitalocêntrico. Isso pode ser exemplificado, em relação à terapia nutricional, quando se observa que é uma prática historicamente reconhecida e realizada em âmbito hospitalar, em que são prescritas fórmulas nutricionais industrializadas. Tais prescrições acabam sendo mantidas após a alta hospitalar por diversos motivos, entre eles: a ausência de organização para continuidade do cuidado na RAS, como inexistência de protocolos e linhas de cuidado, bem como da disponibilidade dos recursos terapêuticos necessários; insegurança ou falta de conhecimento dos profissionais dos outros pontos de atenção para reavaliar a conduta prescrita e propor intervenções coerentes com as condições de vida do indivíduo fora do ambiente hospitalar. Além disso, faltam evidências científicas que apoiem a tomada de decisão dos profissionais em relação à melhor conduta dietética, seja com fórmulas nutricionais industrializadas ou formuladas com alimentos e produtos alimentícios.

Além do sistema fragmentado, traduzido na ainda pouca organização em regionais de saúde e na falta de articulação entre os pontos de atenção da RAS, e da supremacia do modelo hospitalocêntrico, o subfinanciamento e a fragilidade da gestão do SUS também são questões a serem superadas.

Embora ainda existam muitos desafios, não se pode falar em omissão ou descompromisso do Estado com a garantia do direito à saúde, tendo em vista que também têm sido observados inúmeros avanços, como destacado no artigo de Clarice Petramale. Nesse sentido, além dos progressos já apontados por Petramale, destacamos a priorização da atenção básica, inclusive em termos financeiros, nos últimos anos, objetivando garantir o acesso integral ao sistema de saúde com qualidade e resolutividade. Para tanto, houve ampliação do provimento e qualificação de profissionais da saúde, expansão da atenção domiciliar, além de aumento da cobertura populacional da atenção básica, em especial, da Estratégia Saúde da Família, o que gerou impactos na redução da mortalidade por doenças cardiovasculares e hospitalização por essas doenças ${ }^{9}$ e da mortalidade infantil, ${ }^{9}$ superando o Objetivo de Desenvolvimento do Milênio relacionado à redução da mortalidade na infância com quatro anos de antecedência. 
No tocante às ações direcionadas ao público infantil, ressaltamos que o Brasil possui diversas iniciativas que priorizam a atenção integral à criança e abrangem desde a garantia de renda para suas famílias até acesso a serviços de saúde e educação. Assim, em relação à saúde da criança, o Ministério da Saúde, as Secretarias Estaduais e Municipais de Saúde desenvolvem ações de qualificação de profissionais da saúde para atenção humanizada ao recém-nascido e aprimoramento das práticas de cuidado ao parto e puerpério, sendo adotadas as Boas Práticas de Atenção ao Parto e Nascimento, recomendadas pela Organização Mundial da Saúde (OMS). São realizadas, ainda, ações de promoção do aleitamento materno e alimentação complementar saudável, prevenção e controle de carências nutricionais, como hipovitaminose A, anemia e desnutrição, e incentivo à realização de ações de promoção da saúde e acompanhamento nutricional pelas equipes de saúde nas creches e escolas.

Para finalizar, concordamos com Araújo e Bellato, que trazem a discussão de que demandas judiciais servem de alerta para a elaboração de políticas públicas e organização de práticas que visem a promoção, proteção e recuperação da saúde dessa população. Nesse sentido, com base nas demandas judiciais e ainda no contexto de avanços do SUS nos últimos anos, o Ministério da Saúde colocou em consulta pública uma portaria que trata das diretrizes para organização da Terapia Nutricional na Rede de Atenção à Saúde, e outra que atualiza os critérios para credenciamento e habilitação de serviços de Terapia Nutricional em âmbito hospitalar. Ainda estão sendo discutidos a linha de cuidado integral e o Protocolo Clínico e Diretrizes Terapêuticas para crianças com Alergia à Proteína do Leite de Vaca, doença que gera maior demanda por fórmulas nutricionais por via judicial, como demonstrado em nosso artigo.

Além disso, será publicado o Caderno de Atenção Domiciliar sobre Cuidados em Terapia Nutricional, com o objetivo de apoiar as equipes de atenção básica e de atenção domiciliar, bem como gestores do SUS, para a organização e a oferta dos cuidados em Terapia Nutricional no âmbito domiciliar. Da mesma forma, este suplemento temático "Atenção às Necessidades Alimentares Especiais no SUS", contribui para divulgação de evidências científicas e experiências sobre o tema, subsidiando gestores e profissionais para organização e oferta do cuidado às pessoas com necessidades alimentares especiais no SUS. 


\section{Referências}

1. Diniz D, Machado TRC, Penalva J. A judicialização da saúde no Distrito Federal, Brasil. Cienc. Saúde Coletiva 2014; 19(2):591-198.

2. Edais VL, Ventura M, Sant’ana JMB, Figueiredo TA, Souza VR, Simas L. Caracterização de demandas judiciais de fornecimento de medicamentos “essenciais" no Estado do Rio de Janeiro, Brasil. Cad. Saude Publica 2010; 26(3):461-471.

3. Penalva J. Judicialização do direito à saúde: o caso do Distrito Federal. Belo Horizonte: Instituto de Bioética, Direitos Humanos e Gênero; 2011.

4. Travassos DV, Ferreira RC, Vargas AMC, Moura RNV, Conceição EMA, Marques DF, et al. Judicialização da Saúde: um estudo de caso de três tribunais brasileiros. Cien. Saúde Coletiva 2013; 18(11):3419-3429.

5. Marques SB. O direito ao acesso universal a medicamentos no Brasil: diálogos entre o direito, a política e a técnica médica [tese]. São Paulo: Universidade de São Paulo; 2011.

6. Araujo LM, Fraga AJA, Neta AMA, Souza LRB. Judicialização da saúde: uma revisão da literatura. Rev. Enf. UFPI 2013; 2(2):49-54.

7. Moodie R, Stuckler D, Monteiro C, Sheron N, Neal B, Thamarangsi T, Lincoln P, Casswell. Profits and pandemics: prevention of harmful effects of tobacco, alcohol, and ultra-processed food and drink industries. The Lancet. 2013; 381 (9867): 670-9. Acesso em: 08 jul 2014. Disponível em: <http:// www.thelancet.com/journals/lancet/article/PIIS0140-6736(12)62089-3/fulltext>.

8. Rasella D, Harhay MO, Pamponet ML, Aquino R, Barreto ML. Impact of primary health care on mortality from heart and cerebrovascular diseases in Brazil: a nationwide analysis of longitudinal data. BMJ. 2014; 349, g4014. Acesso em: 07 jul 2014. Disponível em: < http://www.bmj.com/content/349/ bmj.g4014.full.pdf+html>.

9. Aquino R, Oliveira NF, Barreto ML. Impacto of the family health program on infant mortality in Brazilian municipalities. AJPH. 2009; 99 (1).

Recebido: 10/7/2014 
